\title{
BALANCED \\ DISTRIBUTION-ENERGY INEQUALITIES AND RELATED ENTROPY BOUNDS
}

\author{
MICHEL RUMIN
}

\begin{abstract}
Let $A$ be a self-adjoint operator acting over a space $X$ endowed with a partition. We give lower bounds on the energy of a mixed state $\rho$ from its distribution in the partition and the spectral density of $A$. These bounds improve with the refinement of the partition, and generalize inequalities by Li-Yau and Lieb-Thirring for the Laplacian in $\mathbb{R}^{n}$. They imply an uncertainty principle, giving a lower bound on the sum of the spatial entropy of $\rho$, as seen from $X$, and some spectral entropy, with respect to its energy distribution. On $\mathbb{R}^{n}$, this yields lower bounds on the sum of the entropy of the densities of $\rho$ and its Fourier transform. A general log-Sobolev inequality is also shown. It holds on mixed states, without Markovian or positivity assumption on $A$.
\end{abstract}

\section{INTRODUCTION AND MAIN RESULTS}

Let $(X, \mu)$ be a $\sigma$-finite measure space, $V$ a separable Hilbert space and $A$ a self-adjoint operator acting on

$$
\mathcal{H}=L^{2}(X, V)=L^{2}(X, \mu) \otimes V .
$$

The inequalities we will consider concern mixed states, that is positive trace class operators on $\mathcal{H}$. From the quantum-mechanical viewpoint, they are positive linear combination of pure states, which are the orthogonal projections on functions in $\mathcal{H}$; see [8, §23] or [9]. More precisely, as in $\|7\|$, we are looking for integral controls on the density of a state $\rho$ from its energy given by the trace

$$
\mathcal{E}(\rho)=\tau(\rho A) .
$$

The density function of the state, or more generally of a bounded positive operator $P$ on $\mathcal{H}$, is a notion that extends the restriction to the diagonal of $X$ of the $V$-trace of the kernel of $P$. It may be defined as follows (see e.g. [7, §1.2]): given a measurable set $\Omega \subset X$, the trace

$$
\nu_{P}(\Omega)=\tau\left(\chi_{\Omega} P \chi_{\Omega}\right)=\tau\left(P^{1 / 2} \chi_{\Omega} P^{1 / 2}\right)
$$

defines a measure on $X$. For any Hilbert basis $\left(e_{i}\right)$ of $\mathcal{H}$, it holds that

$$
\nu_{P}(\Omega)=\int_{\Omega} D \nu_{P}(x) d \mu(x) \quad \text { where } \quad D \nu_{P}(x)=\sum_{i}\left\|\left(P^{1 / 2} e_{i}\right)(x)\right\|_{V}^{2}
$$

is called the density function of $P$. For instance, in the case of a pure state $P=\pi_{f}$ with $\|f\|_{\mathcal{H}}=$ 1, one has $D \nu_{P}(x)=\|f(x)\|_{V}^{2}$. Also, when $V$ is finite dimensional, as for operators acting on

Date: June 1, 2018.

2000 Mathematics Subject Classification. 58J50, 47B06, 46E35, 35P20, 94A17.

Key words and phrases. Lieb-Thirring inequality, entropy, uncertainty principle, log-Sobolev. 
scalar valued functions, it turns out that $D \nu_{P}$ is bounded if and only if $P$ is ultracontractive from $L^{1}(X)$ to $L^{\infty}(X)$ with

$$
\|P\|_{1, \infty} \leq D(P)=\operatorname{supess} D \nu_{P}(x) \leq(\operatorname{dim} V)\|P\|_{1, \infty},
$$

see e.g. [0, Prop. 1.4].

The inequalities studied here rely on the knowledge of the spectral measure associated to $A$. It is defined as follows.

Definition 1.1. Let $A$ be a self-adjoint operator on $\mathcal{H}$ and consider the spectral projections $\Pi_{\lambda}=\Pi_{-\infty, \lambda[}(A)$. We define the spectral measure of a measurable set $\Omega \subset X$ by

$$
F_{\Omega}(\lambda)=\nu_{\Pi_{\lambda}}(\Omega)=\tau\left(\Pi_{\lambda} \chi_{\Omega} \Pi_{\lambda}\right)
$$

and the spectral density function by

$$
F_{x}(\lambda)=D \nu_{\Pi_{\lambda}}(x) .
$$

These functions are positive increasing (in the large sense) and left continuous. In the sequel, if $\varphi: \mathbb{R} \rightarrow \mathbb{R}^{+}$is an increasing function, and $y \geq 0$, we will set

$$
\varphi^{-1}(y)=\sup \{x \in \mathbb{R} \mid \varphi(x) \leq y\} \in[-\infty,+\infty] .
$$

It is a pseudo-inverse of $\varphi$, and right continuous when finite.

1.1. Energy of a confined state and spectral bounds. Our first purpose is to give an inequality between the trace of a state supported in a domain $\Omega$ and its energy.

Theorem 1.2. Let $A$ be a self-adjoint operator acting on $\mathcal{H}=L^{2}(X, V)$, and let $\rho$ be a non-zero state (positive trace class operator) supported in a set $\Omega \subset X$. Suppose that

$$
\mathcal{E}^{+}(\rho)=\tau\left(\rho^{1 / 2} \max (A, 0) \rho^{1 / 2}\right) \text { is finite. }
$$

Then the integral involved below has a finite positive part and it holds that

$$
\|\rho\|_{\infty} \varphi_{\Omega}\left(\frac{\tau(\rho)}{\|\rho\|_{\infty}}\right) \leq \mathcal{E}(\rho)
$$

where $\varphi_{\Omega}(y)=\int_{0}^{y} F_{\Omega}^{-1}(u) d u$ and $\|\rho\|_{\infty}$ denotes the $L^{2}-L^{2}$ norm of $\rho$.

This sharpens and extends Theorem 1.7 in [7], restricted there to positive operators. When applied to projections onto $N$-dimensional spaces $L$ of functions supported in $\Omega$, Theorem 1.2 gives a lower bound on the sum of the $N$-first Dirichlet eigenvalues of $\mathcal{E}$ in $\Omega$, namely

$$
\varphi_{\Omega}(N) \leq \sum_{k=1}^{N} \lambda_{k}(\Omega) \leq \mathcal{E}\left(\Pi_{L}\right) .
$$

Here the Dirichlet spectrum is defined using the min-max principle

$$
\lambda_{n}(\Omega)=\inf _{L \in \mathcal{L}_{n}} \max _{f \in L}\left(\mathcal{E}(f) /\|f\|_{2}^{2}\right) \quad \text { with } \quad \mathcal{L}_{n}=\{\operatorname{supp} L \subset \Omega \mid \operatorname{dim} L=n\} .
$$

As we shall see in $\$ 2.2$, this leads in the case of the Laplacian in $\mathbb{R}^{n}$ to inequalities due to Berezin and Li-Yau (沺 or 䏤, Thm. 12.3]); and which are known to be sharp in the semiclassical limit, i.e. when $N$ goes to $\infty$. Also (7) provides the following controls of the whole Dirichlet spectral distribution in $\Omega$. 
Corollary 1.3. Let $A$ and $\Omega$ as above, and let

$$
N_{\Omega}(\lambda)=\sup \{\operatorname{dim} V \mid \operatorname{supp} V \subset \Omega \text { and } \mathcal{E} \leq \lambda \text { on } V\}
$$

denotes the Dirichlet spectral distribution function of $A$ in $\Omega$. Then one has

$$
\varphi_{\Omega}\left(N_{\Omega}(\lambda)\right) \leq \lambda N_{\Omega}(\lambda) .
$$

If moreover $A$ is positive, then

$$
N_{\Omega}(\lambda) \leq 2 F_{\Omega}(2 \lambda)
$$

Hence in the positive case, the confined spectral distribution in $\Omega$ is controlled by twice the free spectral density seen from $\Omega$ at twice energy level, i.e. by $F_{\Omega}(2 \lambda)=\tau\left(\chi_{\Omega} \Pi_{2 \lambda}\right)$. Indeed there, $\Pi_{2 \lambda}$ is the free (or unconstrained) spectral space of $A$ on the whole $X$.

One feature of the sharpness of inequalities like (6) or (8), that will be used in their proofs, lies in the fact they stay equivalent under an energy shift of $A$ in $A+k$. Indeed, one has then

$$
F_{\Omega}(\lambda) \rightarrow F_{\Omega}(\lambda-k) \text { thus } F_{\Omega}^{-1} \rightarrow F_{\Omega}^{-1}+k \quad \text { and } \quad \varphi_{\Omega}(y) \rightarrow \varphi_{\Omega}(y)+k y .
$$

Hence both sides of (6) shift by $k \tau(\rho)$, while (8) stays unchanged up to a parameter shift. This implies in particular that one can't improve (6) or (8) by a fixed multiplicative factor for any (even positive) operator and state. Indeed, suppose that for any positive operator and state it holds

$$
(1+\varepsilon)\|\rho\|_{\infty} \varphi_{\Omega}\left(\tau(\rho) /\|\rho\|_{\infty}\right) \leq \mathcal{E}_{A}(\rho)
$$

Then one would get by a positive energy shift $A \rightarrow A+k$ that

$$
0 \leq(1+\varepsilon)\|\rho\|_{\infty} \varphi_{\Omega}\left(\tau(\rho) /\|\rho\|_{\infty}\right) \leq \mathcal{E}_{A-k \varepsilon}(\rho)<0
$$

for $k$ large enough. Of course another stronger inequality than (6) may hold however.

In the sequel, we shall say that an inequality is balanced if, like (6) or (8), it stays equivalent through energy shift. None of the inequalities given in $[\nabla]$ is balanced; that precludes them to hold for operators of indefinite sign.

1.2. A balanced Lieb-Thirring inequality. We now state a version of (6), that gives lower bounds on $\mathcal{E}(\rho)$ knowing the distribution of the state in a partition of $X$ into $\sqcup_{i} \Omega_{i}$, i.e. given $\nu_{\rho}\left(\Omega_{i}\right)=\tau\left(\chi_{\Omega_{i}} \rho \chi_{\Omega_{i}}\right)$.

Theorem 1.4. Let $A$ be a self-adjoint operator on $\mathcal{H}=L^{2}(X, V)$, and $\rho$ a non-zero state such that $\mathcal{E}^{+}(\rho)$ is finite. Let $\Omega_{i}$ be a measurable partition of $X$.

- Then the sums and integral involved below have a finite positive part, and it holds that

$$
H_{\Omega_{i}}(\rho)=\|\rho\|_{\infty} \sum_{i} \psi_{\Omega_{i}}\left(\frac{\nu_{\rho}\left(\Omega_{i}\right)}{\|\rho\|_{\infty}}\right) \leq H(\rho)=\|\rho\|_{\infty} \int_{X} \psi_{x}\left(\frac{D \nu_{\rho}(x)}{\|\rho\|_{\infty}}\right) d \mu(x) \leq \mathcal{E}(\rho),
$$

where

$$
\psi_{\Omega_{i}}(y)=\int_{0}^{1} \varphi_{\Omega_{i}, t}(y) d t \quad \text { with } \quad \varphi_{\Omega_{i}, t}(y)=\int_{0}^{y} F_{\Omega}^{-1}\left(t^{2} u\right) d u
$$

and similarly

$$
\psi_{x}(y)=\int_{0}^{1} \varphi_{x, t}(y) d t \quad \text { with } \quad \varphi_{x, t}(y)=\int_{0}^{y} F_{x}^{-1}\left(t^{2} u\right) d u
$$


- Moreover if $\Omega_{i}^{\prime}$ is a finer partition of $X$ than $\Omega_{i}$, then $H_{\Omega_{i}}(\rho) \leq H_{\Omega_{i}^{\prime}}(\rho)$.

These balanced inequalities improve the unbalanced ones given in [0, Thm. 1.6-1.7] for positive operators. They extend an inequality due to Lieb and Thirring in the case of the Laplacian on $\mathbb{R}^{n}$; see $[6]$, [5, Thm. 12.5] and $\$ 3.3$.

To clarify its relation with the previous result, we first remark that since $F_{\Omega}^{-1}$ is increasing, one has

$$
\psi_{\Omega} \leq \varphi_{\Omega}=\varphi_{\Omega, 1} .
$$

Hence if the state is confined in a single domain $\Omega$ of the partition, the bound (6) is stronger than $H_{\Omega}(\rho) \leq \mathcal{E}(\rho)$ in (10). Conversely, we will see in 3.1 that if $A$ is positive, one has

$$
\varphi_{\Omega}\left(\frac{x}{2}\right) \leq \psi_{\Omega}(x),
$$

thus (10) in the confined case actually gives $\|\rho\|_{\infty} \varphi_{\Omega}\left(\frac{\nu_{\rho}(\Omega)}{2\|\rho\|_{\infty}}\right) \leq \mathcal{E}(\rho)$, close to (6), but weaker.

From the quantum-mechanical viewpoint, (10) gives a lower bound on the energy that had a state $\rho$ before the measure of its distribution in the partition, given by $\nu_{\rho}\left(\Omega_{i}\right)=\tau\left(\chi_{\Omega_{i}} \rho \chi_{\Omega_{i}}\right)$, is performed. Equivalently, one gets an a priori control, through $H_{\Omega_{i}}(\rho)$, on the possible outcomes of a measure of the distribution of a state of known energy, before this measure is done. Indeed, in quantum physics (see e.g. [8, 91), an actual measure of this distribution collapses $\rho$ into

$$
\widetilde{\rho}=\sum_{i} \chi_{\Omega_{i}} \rho \chi_{\Omega_{i}},
$$

which is a sum of localized states $\rho_{i}$ in $\Omega_{i}$. By (6) and convexity of $\varphi_{\Omega_{i}}$, one has then

$$
\|\widetilde{\rho}\|_{\infty} \sum_{i} \varphi_{\Omega_{i}}\left(\frac{\left.\nu_{\rho}\left(\Omega_{i}\right)\right)}{\|\widetilde{\rho}\|_{\infty}}\right) \leq \sum_{i}\left\|\rho_{i}\right\|_{\infty} \varphi_{\Omega_{i}}\left(\frac{\left.\nu_{\rho}\left(\Omega_{i}\right)\right)}{\left\|\rho_{i}\right\|_{\infty}}\right) \leq \sum_{i} \mathcal{E}\left(\rho_{i}\right)=\mathcal{E}(\widetilde{\rho}) .
$$

This is stronger than (10) by (13), but applies only to collapsed states.

The monotonicity of $H_{\Omega_{i}}(\rho)$ in the partition makes it behave like an information quantity on the state. It increases with a finer knowledge of the distribution of $\rho$, and is dominated by the continuous integral $H(\rho)$ associated to the "infinitesimal" distribution of $\rho$. Actually these inequalities imply other information-type inequalities like entropy bounds, as we see now.

1.3. Spatial versus spectral entropy and uncertainty principle. One interesting feature of the Lieb-Thirring inequality (10) lies in its simple behaviour under the change of $A$ into $f(A)$ for an increasing right continuous function $f$. Indeed, one has $\Pi_{f(A)}(]-\infty, \lambda[) \subset$ $\Pi_{A}(]-\infty, f^{-1}(\lambda)[)$, and thus for the spectral measures

$$
F_{f(A), \Omega}(\lambda) \leq F_{A, \Omega} \circ f^{-1}(\lambda),
$$

This allows to change the integrals $H(\rho)$ in (10) into many expressions, while using the corresponding energy $\mathcal{E}_{f(A)}(\rho)=\tau(f(A) \rho)$.

An attractive choice is to use $\ln F_{A}^{+}(A)$, where $F_{A}^{+}(\lambda)$ is the right limit of

$$
F_{A}(\lambda)=\operatorname{supess}_{x} F_{A, x}(\lambda) .
$$

For this application, it is crucial that (10) holds for non-positive operator, since $\ln F_{A}^{+}(A)$ is not positive in general. This leads to entropy bounds. 
Theorem 1.5. Let $A$ be a self-adjoint operator and $\rho$ a state such that $\mathcal{E}_{\ln F_{A}^{+}(A)}^{+}(\rho)$ is finite. Then the integral $S_{\mu}(\rho)$ below has a finite negative part and it holds that

$$
S_{\lambda}(\rho)+S_{\mu}(\rho) \geq 0
$$

where

$$
S_{\lambda}(\rho)=\mathcal{E}_{\ln F_{A}^{+}(A)}(\rho) \quad \text { and } \quad S_{\mu}(\rho)=-\int_{X} \ln \left(\frac{D \nu_{\rho}(x)}{\|\rho\|_{\infty}}\right) d \nu_{\rho}(x)+3 \tau(\rho) .
$$

The quantity $S_{\mu}(\rho)$ is related to the "spatial entropy" of the state $\rho$, as seen from the measure space $X$. Actually,

$$
-S_{\mu}(\rho)+\tau(\rho)\left(\ln \|\rho\|_{\infty}+3\right)=\int_{X} \ln \left(\frac{d \nu_{\rho}}{d \mu}\right) d \nu_{\rho}=D_{K L}\left(\nu_{\rho} \| \mu\right)
$$

is the Kullback-Leibler divergence from $\nu_{\rho}$ to $\mu$, or relative entropy of $\nu_{\rho}$ to $\mu$. On the other hand,

$$
S_{\lambda}(\rho)=\tau\left(\ln F_{A}^{+}(A) \rho\right)=\int_{\mathbb{R}} \ln F_{A}^{+}(\lambda) d \tau\left(\rho \Pi_{\lambda}\right),
$$

deals with the "spectral entropy" of $\rho$, as seen from distribution within the spectrum of $A$. Indeed $\ln F_{A}^{+}(\lambda)$ is an analytical ersatz for $\ln \operatorname{dim} E_{\lambda}$ with $E_{\lambda}=E_{]-\infty, \lambda]}(A)$. Namely, for invariant operators acting on groups, one has $F_{A}^{+}(\lambda)=\operatorname{dim}_{\Gamma}\left(E_{\lambda}\right)=\tau_{\Gamma}\left(\Pi_{]-\infty, \lambda]}(A)\right)$ with the notion of von Neumann's $\Gamma$-dimension; see e.g. [7], §1].

This spectral entropy and the inequality (17) have a striking property: they are invariant under the change of $A$ into $f(A)$ for any increasing homeomorphism $f$ of $\mathbb{R}$. Indeed the operator $F_{A}^{+}(A)$ stays unchanged under such transforms, since they give equality in (16). Thus, the spectral entropy is not sensitive to the actual energy levels; it depends only on the ordered set $\left\{\Pi_{\lambda}\right\}$, not its parametrization.

The quantities $S_{\mu, \lambda}(\rho)$ measure the indeterminacy in position and energy of the state. They decrease respectively when $\rho$ is concentrated in a set of small measure, or in small energies. Notice that in the general case, if $X$ is not discrete and $\mu(X)$ infinite, neither $S_{\mu}(\rho)$ nor $S_{\lambda}(\rho)$ are bounded from below. Still, the lower bound for their sum in (17) means that the state can't be arbitrarily localized both in position and energy. This may be seen as a general statement of the uncertainty principle from the entropy viewpoint.

1.4. Fourier transform and entropy. As an illustration of the previous result, we consider a state $\rho$ on $X=\mathbb{R}^{n}$, together with its Fourier transform $\widehat{\rho}$, defined by $\widehat{\rho}(\widehat{f})=\widehat{\rho(f)}$. We shall see, by optimizing the choice of $A$ in (17), the following bound on the sum of the entropy of the density of $\rho$ and the entropy of the distribution of its Fourier transform.

Theorem 1.6. Let vol* be the measure $d^{*} \xi=(2 \pi)^{-n} d \xi$ on $\mathbb{R}^{n}$, and $\rho$ as above. Consider the distribution function of $\nu_{\widehat{\rho}}$ relatively to $d^{*} \xi$

$$
F_{\widehat{\rho}}(y)=\operatorname{vol}^{*}\left(\left\{\xi \in \mathbb{R}^{n} \mid \frac{d \nu_{\widehat{\rho}}}{d^{*} \xi}(\xi) \geq y\right\}\right) .
$$

Suppose that the positive part of

$$
S_{F}(\widehat{\rho})=\int_{0}^{+\infty} \ln \left(F_{\widehat{\rho}}(y)\right) F_{\widehat{\rho}}(y) d y
$$


is finite. Then the negative part of

$$
S_{x}(\rho)=-\int_{\mathbb{R}^{n}} \ln \left(\frac{d \nu_{\rho}}{d x}\right) d \nu_{\rho}(x)
$$

is finite and it holds that

$$
S_{x}(\rho)+S_{F}(\widehat{\rho}) \geq-\tau(\rho)\left(2+\ln \|\rho\|_{\infty}\right) .
$$

This gives an operator free version of the classical uncertainty principle stating that a function (pure state) can't be both arbitrarily localized in position and momentum. As will be seen in $\$ 4.3$, the bound (21) is equivalent to the previous one (17), with $A=\Delta$, for states with spherical density, but improves it on anisotropic ones.

Still, the inequality (21) is not symmetric in the roles of $\rho$ and its Fourier transform $\widehat{\rho}$; because two kinds of entropies are used at the space and frequency sides. However it implies the following symmetric inequality.

Corollary 1.7. It holds that

$$
S_{x}(\rho)+S_{\xi}(\widehat{\rho})=-\int_{\mathbb{R}^{n}} \ln \left(\frac{d \nu_{\rho}}{d x}\right) d \nu_{\rho}-\int_{\mathbb{R}^{n}} \ln \left(\frac{d \nu_{\widehat{\rho}}}{d^{*} \xi}\right) d \nu_{\widehat{\rho}} \geq-\tau(\rho)\left(\ln \tau(\rho)+\ln \|\rho\|_{\infty}\right),
$$

provided the positive part of one of these integrals is finite.

This inequality has a better general behaviour than (21); namely it is additive on tensor products of trace one states. Moreover, at least on projections on finite dimensional spaces, the lower bound is related to von Neumann's proper entropy of $\rho$, defined by $S(\rho)=-\tau(\rho \ln \rho)$; as discussed in $\$ 4.5$.

1.5. Log-Sobolev inequalities. In the general setting, we finally observe that Theorem 1.5 is also related to more classical log-Sobolev inequalities, as stated for instance in [3] or |⿰氵⿹丁口⿹丁口 the Laplacian. Namely, applying Jensen inequality on the spectral entropy in (17) leads to the following entropy-energy bound.

Corollary 1.8. Let $A$ be a self-adjoint operator and $\rho$ a state such that $\mathcal{E}_{A}^{+}(\rho)$ is finite and $\tau(\rho)=1$. Then it holds that

$$
-S_{\mu}(\rho) \leq\left(\ln F_{A}\right)^{c}(\mathcal{E}(\rho))
$$

where $\left(\ln F_{A}\right)^{c}$ is the concave hull of $\ln F_{A}$.

This improves and extends Theorem 1.9 in [|]|, proved there for positive operators and with a larger energy term. The inequality (23) is balanced and even invariant under an affine rescaling of energy $A \rightarrow k_{1} A+k_{2}$ with $k_{1}>0$. It is also equivalent to the family of parametric log-Sobolev inequalities

$$
-S_{\mu}(\rho) \leq m(t) \tau(\rho)+t \mathcal{E}(\rho)
$$

where $m(t)=\inf _{\lambda \geq 0}(\ln F(\lambda)-t \lambda)$ is minus the concave-Legendre transform of $\ln F$. Such inequalities actually hold on mixed states, without Markovian or positivity assumption on $A$. 


\section{The CONFINED STATES INEQUALities}

2.1. Proof of Theorem 1.2. We first show Theorem 1.2 for positive operator, and use after the invariance through energy shift to extend it in the general case.

The proof in the positive case is actually an improvement of an argument given in [7, §3.1]. Let $\Pi_{\geq \lambda}=\Pi_{[\lambda,+\infty[}(A)=\mathrm{Id}-\Pi_{\lambda}$. We observe that

$$
\mathcal{E}(\rho)=\tau\left(\rho^{1 / 2} A \rho^{1 / 2}\right)=\int_{0}^{+\infty} \tau\left(\rho^{1 / 2} \Pi_{\geq \lambda} \rho^{1 / 2}\right) d \lambda .
$$

Since supp $\rho \subset \Omega$, one has $\rho \leq\|\rho\|_{\infty} \chi_{\Omega}$. Hence, assuming by homogeneity in $\rho$ that $\|\rho\|_{\infty}=1$ in the sequel, it holds that

$$
\begin{aligned}
\tau\left(\rho^{1 / 2} \Pi_{\geq \lambda} \rho^{1 / 2}\right) & =\tau(\rho)-\tau\left(\rho^{1 / 2} \Pi_{\lambda} \rho^{1 / 2}\right)=\tau(\rho)-\tau\left(\Pi_{\lambda} \rho \Pi_{\lambda}\right) \\
& \geq \tau(\rho)-\tau\left(\Pi_{\lambda} \chi_{\Omega} \Pi_{\lambda}\right) \\
& =\tau(\rho)-F_{\Omega}(\lambda) .
\end{aligned}
$$

Using it in (25) for $\lambda<F_{\Omega}^{-1}(\tau(\rho))=\sup \left\{u \mid F_{\Omega}(u) \leq \tau(\rho)\right\}$ yields

$$
\begin{aligned}
\mathcal{E}(\rho) & \geq \int_{0}^{F_{\Omega}^{-1}(\tau(\rho))} \tau\left(\rho^{1 / 2} \Pi_{\geq \lambda} \rho^{1 / 2}\right) d \lambda \\
& \geq \int_{0}^{F_{\Omega}^{-1}(\tau(\rho))}\left(\tau(\rho)-F_{\Omega}(\lambda)\right) d \lambda \\
& =\int_{0}^{F_{\Omega}^{-1}(\tau(\rho))}\left(\int_{F_{\Omega}(\lambda)}^{\tau(\rho)} d u\right) d \lambda=\iint_{\left\{0 \leq F_{\Omega}(\lambda) \leq u \leq \tau(\rho)\right\}} d u d \lambda \\
& =\int_{0}^{\tau(\rho)}\left(\int_{0}^{F_{\Omega}^{-1}(u)} d \lambda\right) d u \\
& =\int_{0}^{\tau(\rho)} F_{\Omega}^{-1}(u) d u=\varphi_{\Omega}(\tau(\rho)),
\end{aligned}
$$

as needed.

For a general self-adjoint operator, we consider $A_{k}=A \Pi_{\geq k}$. By positivity of $A_{k}-k$ and the behaviour of (6) in such a shift, it holds for any $k$ that

$$
\varphi_{A_{k}, \Omega}(\tau(\rho)) \leq \mathcal{E}_{A_{k}}(\rho) .
$$

In particular, for $k=0$, one has $\max \left(F_{A}^{-1}, 0\right) \leq F_{A_{0}}^{-1}$ and thus

$$
\int_{0}^{\tau(\rho)} \max \left(F_{A}^{-1}(u), 0\right) d u \leq \varphi_{A_{0}, \Omega}(\tau(\rho)) \leq \mathcal{E}_{A_{0}}(\rho)=\mathcal{E}^{+}(\rho)<\infty
$$

by hypothesis. Hence the integral $\varphi_{A, \Omega}(\tau(\rho))=\int_{0}^{\tau(\rho)} F_{A, \Omega}^{-1}(u) d u$ makes sense in $[-\infty,+\infty[$. If $\varphi_{A, \Omega}(\tau(\rho))=-\infty$ there is nothing more to prove, and we assume henceforth that $\varphi_{A, \Omega}(\tau(\rho))$ is finite. This implies that the increasing function $F_{A, \Omega}^{-1}(u)$ is finite for $u<\tau(\rho)$. In particular, one has necessarily $F_{A, \Omega}(k)$ finite for $k \ll 0$, and thus by dominated convergence

$$
F_{A, \Omega}(k)=\tau\left(\chi_{\Omega} \Pi_{]-\infty, k[}(A) \chi_{\Omega}\right) \searrow 0 \quad \text { when } \quad k \searrow-\infty .
$$


Since, for $k \leq \lambda$, one has $\Pi_{\lambda}\left(A_{k}\right)=\Pi_{[k, \lambda[}(A)=\Pi_{\lambda}(A)-\Pi_{k}(A)$, it holds that

$$
F_{A_{k}, \Omega}(\lambda)=\max \left(F_{A, \Omega}(\lambda)-F_{A, \Omega}(k), 0\right) .
$$

This leads to $F_{A_{k}, \Omega}^{-1}(u)=F_{A, \Omega}^{-1}\left(u+F_{A, \Omega}(k)\right)$, and finally

$$
\varphi_{A_{k}, \Omega}(y)=\int_{0}^{y} F_{A_{k}, \Omega}^{-1}(u) d u=\int_{0}^{y} F_{A, \Omega}^{-1}\left(u+F_{A, \Omega}(k)\right) d u .
$$

Together with (28) and (27), this shows that $\varphi_{A_{k}, \Omega}(\tau(\rho)) \searrow \varphi_{A, \Omega}(\tau(\rho))$ when $k \searrow-\infty$; by dominated convergence for the positive part, and monotone convergence for the negative one. For the same reasons, one has $\mathcal{E}_{A_{k}}(\rho) \searrow \mathcal{E}_{A}(\rho)$ for $k \searrow-\infty$, giving the result by (27).

2.2. Illustration in $\mathbb{R}^{n}$. As a first illustration of the previous result, we consider the case of the Laplacian on $X=\mathbb{R}^{n}$. By group invariance, the density $F_{x}(\lambda)$ is a constant given by the value at 0 of the kernel of $\Pi_{\lambda}$. To compute it, we remark that the spectral spaces $E_{\Delta}(\lambda)$ are functions whose Fourier transforms are supported in the ball $B_{n}\left(0, \lambda^{1 / 2}\right)$. It follows easily (see e.g. [7. §4.2]) that

$$
F_{x}(\lambda)=\widehat{\chi}_{B_{n}\left(0, \lambda^{1 / 2}\right)}(0)=C_{n} \lambda^{n / 2} \quad \text { with } \quad C_{n}=(2 \pi)^{-n} \operatorname{vol}\left(B_{n}(0,1)\right),
$$

and thus

$$
\varphi_{\Omega}(y)=\int_{0}^{y} F_{\Omega}^{-1}(u) d u=\frac{n}{n+2}\left(C_{n} \operatorname{vol}(\Omega)\right)^{-2 / n} y^{1+2 / n} .
$$

Applying Theorem 1.2 to the orthogonal projection $\rho$ on the first $N$ Dirichlet eigenfunctions of $\Delta$ in $\Omega$, yields the following inequality, due to Berezin and Li-Yau (see [4], [5, Thm. 12.3])

$$
\mathcal{E}(\rho)=\sum_{i=1}^{N} \lambda_{i}(\Omega) \geq \frac{n}{n+2}\left(C_{n} \operatorname{vol}(\Omega)\right)^{-2 / n} N^{1+2 / n} .
$$

When $\Omega$ is a domain of finite boundary area, this bound is known to be sharp, up to lower order term in $N$, in the semiclassical limit, i.e. for $N$ goes to $\infty$; see e.g. [5. Thm. 12.11],

2.3. Equality case and bathtub filling. The proof of Theorem 1.2 above shows that $\mathcal{E}(\rho)$ gets smaller and approaches the proposed lower bound $\varphi_{\Omega}(\tau(\rho))$ when:

(1) $\rho$ is the largest possible, i.e close to $\chi_{\Omega}$, on $\Pi_{\lambda}$ for $\lambda<\lambda_{0}=F_{\Omega}^{-1}(\tau(\rho))$;

(2) and $\rho$ is the smallest possible, i.e. close to 0 , on $\Pi_{>\lambda_{0}}$.

That means that $\rho$ has to fill up, or saturate, as much as possible the lower energy levels it can, under the constraint that $\rho \subset \subset \Omega$ and until the volume $\tau(\rho)$ is reached. This kind of idea, clear from the physical viewpoint, is actually quite similar to the bathtub principle used in the proof of Li-Yau inequality (32) given by Lieb and Loss in [5, Theorem 12.3].

In general, one can't have equality in (6) unless $\rho$ is pinched between $\Pi_{\lambda_{0}}=\Pi_{-\infty, \lambda_{0}[}(A)$ and $\Pi_{\lambda_{0}^{+}}=\Pi_{\left.]-\infty, \lambda_{0}\right]}(A)$ and supported in $\Omega$. Hence, if the spectral spaces of $A$ are not confined in a proper subspace $\Omega$ of the ambient space $X$, the only remaining possibility is to take $\Omega=X$ itself. This requires of course that $\operatorname{dim} E_{\lambda_{0}^{-}}=\tau\left(\Pi_{\lambda_{0}^{-}}\right) \leq \tau(\rho)$ be finite. 
2.4. Asymptotic sharpness and amenability. One can go beyond the previous equality case and describe situations with $X$ infinite and where (6) is asymptotically sharp. Given $\lambda$ and $\Omega$, one considers the two states

$$
\rho_{\Omega}=\chi_{\Omega} \Pi_{\lambda} \chi_{\Omega} \quad \text { and } \quad \widetilde{\rho}_{\Omega}=\Pi_{\lambda} \chi_{\Omega} \Pi_{\lambda} .
$$

Notice that $\rho_{\Omega}$ is confined in $\Omega$ while $\widetilde{\rho}_{\Omega}$ is not. Still, one has $\tau\left(\rho_{\Omega}\right)=\tau\left(\widetilde{\rho}_{\Omega}\right)=F_{\Omega}(\lambda)$ and we claim that

$$
\varphi_{\Omega}\left(\tau\left(\rho_{\Omega}\right)\right)=\varphi_{\Omega}\left(F_{\Omega}(\lambda)\right)=\int_{]-\infty, \lambda[} u d F_{\Omega}(u)=\mathcal{E}\left(\widetilde{\rho}_{\Omega}\right)
$$

if this converges. To see this we proceed as in (26), assuming first that $A$ is positive. One finds

$$
\begin{aligned}
\varphi_{\Omega}\left(F_{\Omega}(\lambda)\right) & =\int_{0}^{F_{\Omega}^{-1}\left(F_{\Omega}(\lambda)\right)}\left(F_{\Omega}(\lambda)-F_{\Omega}(u)\right) d u \\
& =\int_{0}^{\lambda}\left(F_{\Omega}(\lambda)-F_{\Omega}(u)\right) d u,
\end{aligned}
$$

since $F_{\Omega}(u)=F_{\Omega}(\lambda)$ for $0 \leq \lambda \leq u \leq F_{\Omega}^{-1}\left(F_{\Omega}(\lambda)\right)$. Thus

$$
\begin{aligned}
\varphi_{\Omega}\left(F_{\Omega}(\lambda)\right) & =\int_{0 \leq u \leq v<\lambda} d F_{\Omega}(v) d u \\
& =\int_{[0, \lambda[} v d F_{\Omega}(v) .
\end{aligned}
$$

The general case follows by energy cut-off and shift as in $\$ 2.1$.

When $\Omega$ is large, $\left\|\widetilde{\rho}_{\Omega}\right\|_{\infty}$ is close to 1 , and (33) means that (6) is sharp for these states $\widetilde{\rho}_{\Omega}$. However they are not confined in $\Omega$. Still $\mathcal{E}\left(\rho_{\Omega}\right)$ may be compared to $\mathcal{E}\left(\widetilde{\rho}_{\Omega}\right)$ in the following situation. If $X$ is a discrete metric space, and $A$ is a bounded local operator, i.e. $A f(x)$ depends only on the value of $f$ in the ball $B(x, r)$, then one has

$$
\begin{aligned}
\left|\mathcal{E}\left(\rho_{\Omega}\right)-\mathcal{E}\left(\widetilde{\rho}_{\Omega}\right)\right| & =\left|\tau\left(A \chi_{\Omega} \Pi_{\lambda} \chi_{\Omega}\right)-\tau\left(A \Pi_{\lambda} \chi_{\Omega} \Pi_{\lambda}\right)\right| \\
& =\left|\tau\left(\Pi_{\lambda} \chi_{\Omega}\left(A \chi_{\Omega}-\chi_{\Omega} A\right)\right)\right| \\
& \leq 2\|A\|_{\infty}\left|\partial_{r} \Omega\right|
\end{aligned}
$$

where $\left|\partial_{r} \Omega\right|$ is the cardinal of $\partial_{r} \Omega=\left\{x \in X \mid d(x, \Omega) \leq r\right.$ and $\left.d\left(x, \Omega^{c}\right) \leq r\right\}$. This leads to the following asymptotic sharpness result for (6).

Proposition 2.1. Let $X=\Gamma$ be a discrete infinite amenable group, endowed with an invariant measure, and let $A$ be a local translation invariant symmetric operator on $X$. Suppose that $\Omega_{n}$ is a Fölner sequence such that $\left|\partial_{r} \Omega_{n}\right| /\left|\Omega_{n}\right| \rightarrow 0$ when $n \rightarrow+\infty$. Set $F=F_{x}$ and $\varphi=\varphi_{x}$ (constant in $x$ ). Then it holds that

$$
\lim _{n \rightarrow+\infty} \mathcal{E}\left(\rho_{\Omega_{n}}\right) /\left|\Omega_{n}\right|=\lim _{n \rightarrow+\infty}\left\|\rho_{\Omega_{n}}\right\|_{\infty} \varphi_{\Omega_{n}}\left(\frac{\tau\left(\rho_{\Omega_{n}}\right)}{\left\|\rho_{\Omega_{n}}\right\|_{\infty}}\right) /\left|\Omega_{n}\right|=\varphi(F(\lambda)) .
$$

This may be seen as the counterpart in the discrete setting to the semiclassical result recalled in $\$ 2.2$; here the sharpness of (6) is achieved on large domains and fixed energy, instead of 
the contrary in the semiclassical limit. This statement applies for instance to the discrete Laplacians on $\ell^{2}$-cochains over amenable coverings of finite simplicial complex.

2.5. Faber-Krahn inequality and the heat technique. We can compare the lower bound on the Dirichlet spectrum, or Faber-Krahn inequality, obtained in (7):

$$
\lambda_{1}(\Omega) \geq \varphi_{\Omega}(1),
$$

to the one shown in [2, Prop. II.2] using a heat kernel technique. Namely, it follows from the Nash inequality given there that if $A$ is a positive operator, one has

$$
\lambda_{1}(\Omega) \geq \theta(\Omega)=\sup _{t>0} \frac{1}{t} \ln \left(\frac{1}{L(t) \mu(\Omega)}\right),
$$

where $L(t)=\left\|e^{-t A}\right\|_{1, \infty}$. This bound is actually weaker than (35), at least on scalar operators. Indeed, by (3), it holds that

$$
\begin{aligned}
L(t) \mu(\Omega) & \geq \nu_{e^{-t A}}(\Omega)=\tau\left(\chi_{\Omega} e^{-t A} \chi_{\Omega}\right) \\
& =\int_{0}^{+\infty} e^{-t \lambda} d F_{\Omega}(\lambda) \\
& \geq \int_{\left[0, F_{\Omega}^{-1}(1)\right]} e^{-t \lambda} d \widetilde{F}_{\Omega}(\lambda)
\end{aligned}
$$

with $\widetilde{F}_{\Omega}(\lambda)=F_{\Omega}(\lambda)$ for $\lambda<F_{\Omega}^{-1}(1)$ and $\widetilde{F}_{\Omega}\left(F_{\Omega}^{-1}(1)\right)=1$. Notice that $0 \leq d \widetilde{F}_{\Omega} \leq d F_{\Omega}$ since $F_{\Omega}\left(F_{\Omega}^{-1}(1)\right) \leq 1 \leq F_{\Omega}\left(F_{\Omega}^{-1}(1)^{+}\right)$by left continuity of $F_{\Omega}$. Then by Jensen,

$$
\begin{aligned}
-\ln (L(t) \mu(\Omega)) & \leq t \int_{\left[0, F_{\Omega}^{-1}(1)\right]} \lambda d \widetilde{F}_{\Omega}(\lambda) \\
& =t \int_{0}^{1}\left(1-F_{\Omega}(\lambda)\right) d u=t \varphi_{\Omega}(1),
\end{aligned}
$$

by (26). This gives $\theta(\Omega) \leq \varphi_{\Omega}(1)$ as claimed.

2.6. Proof of Corollary 1.3. When $A$ is a positive operator, one has for $c \in[0,1]$,

$$
\begin{aligned}
\varphi_{\Omega}(y) & =\int_{0}^{y} F_{\Omega}^{-1}(u) d u \geq \int_{c y}^{y} F_{\Omega}^{-1}(u) d u \\
& \geq(1-c) y F_{\Omega}^{-1}(c y) .
\end{aligned}
$$

Hence (8), that comes from (7), implies

$$
N_{\Omega}(\lambda) \leq \frac{1}{c} F_{\Omega}\left(\frac{\lambda}{1-c}\right),
$$

giving (9) in the case $c=1 / 2$. Unlike (\$) these inequalities are not balanced. If $F_{\Omega}$ is a concave function, one can sharpen (37) into $N_{\Omega}(\lambda) \leq 2 F_{\Omega}(\lambda)$ by Jensen. When $F_{\Omega}(\lambda) / \lambda$ is increasing, for instance when $F_{\Omega}$ is a convex function, one sees easily that $N_{\Omega}(\lambda) \leq F_{\Omega}(2 \lambda)$.

\section{The Balanced LieB-Thirring InEQUaLity}

We now consider Theorem 1.4 and begin with the continuous case. The argument is an improvement of $[7, \S 3.2]$. 
3.1. Proof of $H(\rho) \leq \mathcal{E}(\rho)$. Let $\rho$ be a state, $\Omega$ any measurable set in $X$, and let consider the splitting

$$
\rho^{1 / 2} \chi_{\Omega}=\rho^{1 / 2} \Pi_{\lambda} \chi_{\Omega}+\rho^{1 / 2} \Pi_{\geq \lambda} \chi_{\Omega} .
$$

Using Hilbert-Schmidt norm and assuming by homogeneity that $\left\|\rho^{1 / 2}\right\|_{\infty}=\|\rho\|_{\infty}^{1 / 2}=1$ yield

$$
\begin{aligned}
\left\|\rho^{1 / 2} \chi_{\Omega}\right\|_{H S} & \leq\left\|\rho^{1 / 2} \Pi_{\lambda} \chi_{\Omega}\right\|_{H S}+\left\|\rho^{1 / 2} \Pi_{\geq \lambda} \chi_{\Omega}\right\|_{H S} \\
& \leq\left\|\Pi_{\lambda} \chi_{\Omega}\right\|_{H S}+\left\|\rho^{1 / 2} \Pi_{\geq \lambda} \chi_{\Omega}\right\|_{H S} .
\end{aligned}
$$

Since $\|P\|_{H S}=\tau\left(P^{*} P\right)^{1 / 2}=\tau\left(P P^{*}\right)^{1 / 2}$, one finds by (1) that

$$
\nu_{\rho}(\Omega)^{1 / 2} \leq \nu_{\Pi_{\lambda}}(\Omega)^{1 / 2}+\nu_{\Pi_{\geq \lambda} \rho \Pi_{\geq \lambda}}(\Omega)^{1 / 2} .
$$

This implies a similar inequality almost everywhere at the local level, i.e.

$$
D \nu_{\rho}(x)^{1 / 2} \leq F_{x}(\lambda)^{1 / 2}+D \nu_{\Pi_{\geq \lambda} \rho \Pi_{\geq \lambda}}(x)^{1 / 2} .
$$

Indeed, using (38) on the sets

$$
\Omega_{a, b, c}=\left\{x \in X \mid D \nu_{\rho}(x) \geq a^{2}, F_{x}(\lambda) \leq b^{2} \text { and } D \nu_{\Pi_{>\lambda} \rho \Pi_{>\lambda}}(x) \leq c^{2}\right\}
$$

with $(a, b, c) \in D=\left\{a, b, c \in \mathbb{Q}^{+} \mid a>b+c\right\}$, gives that $\mu\left(\Omega_{a, b, c}\right)=0$. Whence

$$
\{x \in X \mid \text { (39) fails }\}=\bigcup_{D} \Omega_{a, b, c}
$$

is also negligible. The author is grateful to Guy David for suggesting this level set argument.

We now suppose that $A$ is positive, and uses (25),

$$
\begin{aligned}
\mathcal{E}(\rho) & =\int_{0}^{+\infty} \tau\left(\rho^{1 / 2} \Pi_{\geq \lambda} \rho^{1 / 2}\right) d \lambda=\int_{0}^{+\infty} \tau\left(\Pi_{\geq \lambda} \rho \Pi_{\geq \lambda}\right) d \lambda \\
& =\int_{0}^{+\infty} \nu_{\Pi_{\geq \lambda} \rho \Pi_{\geq \lambda}}(X) d \lambda \\
& =\int_{X \times \mathbb{R}^{+}} D \nu_{\Pi_{\geq \lambda} \rho \Pi_{\geq \lambda}}(x) d \mu(x) d \lambda \\
& \geq \int_{\Omega} D \nu_{\Pi_{\geq \lambda} \rho \Pi_{\geq \lambda}}(x) d \mu(x) d \lambda
\end{aligned}
$$

where $\Omega=\left\{(x, \lambda) \in X \times \mathbb{R}^{+} \mid F_{x}(\lambda) \leq D \nu_{\rho}(x)\right\}$. Then, by (39),

$$
\begin{aligned}
\mathcal{E}(\rho) & \geq \int_{\Omega}\left(D \nu_{\rho}(x)^{1 / 2}-F_{x}(\lambda)^{1 / 2}\right)^{2} d \mu(x) d \lambda \\
& =\int_{X} \psi_{x}\left(D \nu_{\rho}(x)\right) d \mu(x)
\end{aligned}
$$

with

$$
\psi_{x}(y)=\int_{0}^{F_{x}^{-1}(y)}\left(y^{1 / 2}-F_{x}(\lambda)^{1 / 2}\right)^{2} d \lambda .
$$

We shall compare this expression to $\varphi_{x}(y)=\int_{0}^{y} F_{x}^{-1}(t) d t$. First, using

$$
\sqrt{y}-\sqrt{u} \geq \sqrt{\frac{y}{2}-u} \text { for } 0 \leq u \leq \frac{y}{2},
$$


and proceeding as in (26), one finds that

$$
\begin{aligned}
\psi_{x}(y) & \geq \int_{0}^{F_{x}^{-1}(y / 2)}\left(\frac{y}{2}-F_{x}(\lambda)\right) d \lambda \\
& =\int_{0}^{y / 2} F_{x}^{-1}(t) d t=\varphi_{x}\left(\frac{y}{2}\right),
\end{aligned}
$$

This shows the comparison (14) claimed for positive operators. For the general expression (12), one uses (41)

$$
\begin{aligned}
\psi_{x}(y) & =\int_{0}^{F_{x}^{-1}(y)} \int_{F_{x}(\lambda)}^{y}\left(u^{1 / 2}-F_{x}(\lambda)^{1 / 2}\right) \frac{d u}{\sqrt{u}} d \lambda \\
& =\int_{0}^{F_{x}^{-1}(y)} \int_{F_{x}(\lambda)}^{y} \int_{F_{x}(\lambda)}^{u} \frac{d v d u d \lambda}{2 \sqrt{u v}} \\
& =\int_{\left\{0 \leq F_{x}(\lambda) \leq v \leq u \leq y\right\}} \frac{d v d u d \lambda}{2 \sqrt{u v}} \\
& =\int_{0}^{y} \int_{0}^{u} \int_{0}^{F_{x}^{-1}(v)} d \lambda \frac{d v}{2 \sqrt{v}} \frac{d u}{\sqrt{u}} \\
& =\int_{0}^{y} \int_{0}^{u} F_{x}^{-1}(v) \frac{d v}{2 \sqrt{v}} \frac{d u}{\sqrt{u}} \\
& =\int_{0}^{y} \int_{0}^{1} F_{x}^{-1}\left(t^{2} u\right) d t d u \\
& =\int_{0}^{1} \varphi_{x, t}(y) d t,
\end{aligned}
$$

with $\varphi_{x, t}(y)=\int_{0}^{y} F_{x}^{-1}\left(t^{2} u\right) d u$ as needed. This shows that $H(\rho) \leq \mathcal{E}(\rho)$ for positive operators.

Remark 3.1. The inequality $H_{\Omega_{i}}(\rho) \leq \mathcal{E}(\rho)$ for partitions can be proved along the same lines; just replacing (40) above by its discrete analogous

$$
\mathcal{E}(\rho) \geq \sum_{i} \int_{0}^{F_{\Omega_{i}}^{-1}\left(\nu_{\rho}\left(\Omega_{i}\right)\right)} \nu_{\Pi_{\lambda} \rho \Pi_{\lambda}}\left(\Omega_{i}\right) d \lambda,
$$

and using (38) in place of (39). Furthermore, the previous computations on $\varphi_{x}$ and $\psi_{x}$ apply on $\varphi_{\Omega_{i}}$ and $\psi_{\Omega_{i}}$ instead.

The case of general (non-positive) operators can be handled as in $\$ 2.1$; using the cut-off $A_{k}=\max (A, k)$ and energy shift in these balanced inequalities. From the positive case, one has

$$
\int_{X} \int_{0}^{D \nu_{\rho}(x)} \int_{0}^{1} \max \left(F_{x}^{-1}\left(t^{2} u\right), 0\right) d t d u d \mu(x) \leq \mathcal{E}^{+}(\rho)<\infty,
$$

Hence $\mathcal{E}^{+}(\rho)$ controls the positive part of the integral $H(\rho)$. Then taking $k \searrow-\infty$ yields the result: by dominated convergence for the positive part and monotone convergence for the negative one. 
3.2. Behaviour of $H_{\Omega_{i}}$ under partition refinement. We shall now prove that

$$
H_{\Omega_{i}} \leq H_{\Omega_{i}^{\prime}} \leq H
$$

if $\Omega_{i}^{\prime}$ is a finer partition of $X$ than $\Omega_{i}$. This will actually follow by integration in $\left.\left.t \in\right] 0,1\right]$ of the parametric inequalities

$$
H_{\Omega_{i}, t} \leq H_{\Omega_{i}^{\prime}, t} \leq H_{t}
$$

where

$$
H_{\Omega_{i}, t}(\rho)=\|\rho\|_{\infty} \sum_{i} \varphi_{\Omega_{i}, t}\left(\frac{\nu_{\rho}\left(\Omega_{i}\right)}{\|\rho\|_{\infty}}\right) \quad \text { and } \quad H_{t}(\rho)=\|\rho\|_{\infty} \int_{X} \varphi_{x, t}\left(\frac{D \nu_{\rho}(x)}{\|\rho\|_{\infty}}\right) d \mu(x) .
$$

Remark 3.2. When $t=1$, we have seen in (15) that these expressions give energy lower bounds of collapsed states, and (42) means they also behave like an information quantity; actually finer than the averaged $H$, but restricted to such states.

We start with the discrete vs. continuous inequality, in the positive case, i.e. $F_{x}(0)=0$, and assume again that $\|\rho\|_{\infty}=1$. Given $t>0$,

$$
\varphi_{x, t}(y)=\int_{0}^{y} F_{x}^{-1}\left(t^{2} u\right) d u \quad \text { and } \quad \varphi_{\Omega_{i}, t}(y)=\int_{0}^{y} F_{\Omega_{i}}^{-1}\left(t^{2} u\right) d u
$$

are convex functions whose Legendre transforms are respectively

$$
\varphi_{x, t}^{*}(z)=\int_{0}^{z} F_{x}(v) \frac{d v}{t^{2}} \quad \text { and } \quad \varphi_{\Omega_{i}, t}^{*}(z)=\int_{0}^{z} F_{\Omega_{i}}(v) \frac{d v}{t^{2}} .
$$

Young's inequality states that for any $y, z \geq 0$

$$
y z \leq \varphi_{x, t}(y)+\varphi_{x, t}^{*}(z) .
$$

Integrating it over $\Omega_{i}$ with $y=D \nu_{\rho}(x)$ yields

$$
\begin{aligned}
z \nu_{\rho}\left(\Omega_{i}\right) & \leq \int_{\Omega_{i}} \varphi_{t, x}\left(D \nu_{\rho}(x)\right) d \mu(x)+\int_{\Omega_{i}} \int_{0}^{z} F_{x}(v) \frac{d v}{t^{2}} d \mu(x) \\
& =\int_{\Omega_{i}} \varphi_{x, t}\left(D \nu_{\rho}(x)\right) d \mu(x)+\varphi_{\Omega_{i}, t}^{*}(z),
\end{aligned}
$$

by Fubini and (5). Then by Legendre duality, one has

$$
\varphi_{\Omega_{i}, t}\left(\nu_{\rho}\left(\Omega_{i}\right)\right)=\sup _{z \geq 0}\left(z \nu_{\rho}\left(\Omega_{i}\right)-\varphi_{\Omega_{i}, t}^{*}(z)\right) \leq \int_{\Omega_{i}} \varphi_{x, t}\left(D \nu_{\rho}(x)\right) d \mu(x) .
$$

This gives $H_{\Omega_{i}, t}(\rho) \leq H_{t}(\rho)$ by summation. The discrete comparison $H_{\Omega_{i}}(\rho) \leq H_{\Omega_{i}^{\prime}}(\rho)$ follows the same lines: just replacing the integration over $\Omega_{i}$ above by the discrete splitting of $\Omega_{i}$ into smaller $\Omega_{j}^{\prime}$.

We now consider the general (non-positive) situation. From $\$ 3.1$, the positive parts of $H_{t}(\Omega)$ and $H_{\Omega_{i}, t}(\rho)$ are finite if $\mathcal{E}^{+}(\rho)$ is. Moreover we shall assume that the negative part of $H_{\Omega_{i}, t}(\rho)$ is finite, or (42) is already satisfied. This implies in particular that $F_{\Omega_{i}}^{-1}(u)>-\infty$ for any $i$ and $u>0$, and thus the functions $F_{\Omega_{i}}(\lambda)=\int_{\Omega_{i}} F_{x}(\lambda) d \mu(x) \searrow 0$ when $\lambda \searrow-\infty$. Whence, fixing an $i$, one has a.e. in $\Omega_{i}$ that $F_{x}(\lambda) \searrow 0$ when $\lambda \searrow-\infty$. We shall now apply (43) to

$$
F_{k, x}(\lambda)=F_{x}(\lambda+k)-F_{x}(k) \quad \text { and } \quad F_{k, \Omega_{i}}(\lambda)=F_{\Omega_{i}}(\lambda+k)-F_{\Omega_{i}}(k) .
$$


This gives

$$
\left.\left.F_{k, x}^{-1}(u)=F_{x}^{-1}\left(u+F_{x}(k)\right)\right)-k \quad \text { and } \quad F_{k, \Omega_{i}}^{-1}(u)=F_{\Omega_{i}}^{-1}\left(u+F_{\Omega_{i}}(k)\right)\right)-k,
$$

and

$$
\int_{0}^{\nu_{\rho}\left(\Omega_{i}\right)} F_{\Omega_{i}}^{-1}\left(t^{2} u+F_{\Omega_{i}}^{-1}(k)\right) d u \leq \int_{\Omega_{i}} \int_{0}^{D \nu_{\rho}(x)} F_{x}^{-1}\left(t^{2} u+F_{x}(k)\right) d u d \mu(x),
$$

leading to the result for $k \searrow-\infty$.

3.3. Illustration in $\mathbb{R}^{n}$. We consider again the case of the Laplacian on $\mathbb{R}^{n}$. From (30), one has

$$
F_{n}^{-1}(u)=C_{n}^{-2 / n} u^{2 / n}=4 \pi \Gamma(1+n / 2)^{2 / n} u^{2 / n},
$$

giving

$$
\psi_{n}(y)=\int_{0}^{1} \int_{0}^{y} F_{n}^{-1}\left(t^{2} u\right) d u d t=D_{n} y^{1+2 / n}
$$

with

$$
D_{n}=\frac{4 \pi}{(1+4 / n)(1+2 / n)} \Gamma(1+n / 2)^{2 / n} .
$$

Thus, if $\rho$ is a projection onto a $N$-dimensional space of orthonormal basis $f_{i}$, (10) reads

$$
D_{n} \int_{\mathbb{R}^{n}}\left(\sum_{i=1}^{N}\left|f_{i}(x)\right|^{2}\right)^{1+2 / n} d x \leq \sum_{i=1}^{N}\left\|\nabla f_{i}\right\|_{2}^{2} .
$$

Such lower bound of the kinetic energy is due to Lieb-Thirring, see [5, Thm. 12.5] or [6], and have applications in quantum mechanics. Notice that similar bounds can also be obtained from (10) for $|\nabla|=\Delta^{1 / 2}$ or the relativistic kinetic energy $P=\left(\Delta+m^{2}\right)^{1 / 2}-m$ (see $\|5\|$ ); replacing $F_{n}^{-1}$ above by $F_{|\nabla|}^{-1}=\left(F_{n}^{-1}\right)^{1 / 2}$ or $F_{P}^{-1}(\lambda)=\left(F_{n}^{-1}+m^{2}\right)^{1 / 2}-m$.

The constant $D_{n}$ given here is quite sharp for large $n$. Indeed, by $[5, \S 12.5]$, the (unknown) best constant has to be smaller than $B_{n}=(1+4 / n) D_{n}$. This follows from the remark that

$$
\varphi_{n}(y)=\int_{0}^{y} F_{n}^{-1}(u) d u=B_{n} y^{1+2 / n} .
$$

Indeed by Jensen inequality (or (42)) and Berezin-Li-Yau inequality (32) one has both

$$
\varphi_{n, \Omega}(N)=\mu(\Omega)^{-2 / n} \varphi_{n}(N) \leq B_{n} \int_{\Omega}\left(\sum_{i=1}^{N}\left|f_{i}(x)\right|^{2}\right)^{1+2 / n} d x \text { and } \sum_{i=1}^{N}\left\|\nabla f_{i}\right\|_{2}^{2},
$$

for functions confined in a domain $\Omega$. As the second inequality is sharp in the semiclassical limit $N \rightarrow+\infty$, the best constant in (44) is smaller than $B_{n}$ as claimed. 


\section{ENTROPY BOUNDS}

4.1. Proof of Theorem 1.5. We deduce the inequality between the spatial and spectral entropies from Theorem 1.5. Consider the functions

$$
F_{A}(\lambda)=\sup _{A, \Omega} \frac{F_{A, \Omega}(\lambda)}{\mu(\Omega)}=\operatorname{supess}_{x} F_{A, x}(\lambda) \quad \text { and } \quad F_{A}^{+}(\lambda)=\lim _{\varepsilon \rightarrow 0^{+}} F_{A}(\lambda+\varepsilon) .
$$

We observe that $F_{A}$ is increasing and left continuous, since the $F_{A, \Omega}$ are, while $F_{A}^{+}$is right continuous. We shall assume that $F_{A}(\lambda)$ is finite for $\lambda \ll 0$, in order that the hypothesis of Theorem 1.5 hold for some state. This implies in particular by dominated convergence that $F_{A}(\lambda) \searrow 0$ when $\lambda \searrow-\infty$. Then by (16), one has

$$
F_{\ln F_{A}^{+}(A)}(\lambda) \leq F_{A} \circ\left(F_{A}^{+}\right)^{-1}\left(e^{\lambda}\right) \leq F_{A} \circ F_{A}^{-1}\left(e^{\lambda}\right) \leq e^{\lambda},
$$

by left continuity of $F_{A}$. Hence by (12), it holds a.e. in $X$ that

$$
\psi_{\ln F_{A}^{+}(A), x}(y) \geq \int_{0}^{1} \int_{0}^{y} \ln \left(t^{2} u\right) d u d t=y \ln y-3 y,
$$

leading to Theorem 1.5 .

4.2. Illustration on $\mathbb{R}^{n}$. We make explicit Theorem 1 1.5 in the case of the Laplacian on $\mathbb{R}^{n}$. Given a state $\rho$, we can express its spectral entropy $S_{\lambda}(\rho)$ using Fourier transform. Suppose that $\rho=\sum_{i} p_{i} \Pi_{f_{i}}$ for orthonormal functions $f_{i}$. Its Fourier transform $\widehat{\rho}$ acts on $L^{2}\left(\mathbb{R}_{\xi}^{n}\right)$ by $\widehat{\rho}(\widehat{f})=\widehat{\rho(f)}$; actually $\widehat{\rho}=\sum_{i} p_{i} \Pi_{\widehat{f}}$ using the Plancherel measure $d^{*} \xi=(2 \pi)^{-n} d \xi$. At the density level, this writes

$$
d \nu_{\rho}(x)=\sum_{i} p_{i}\left|f_{i}(x)\right|^{2} d x \quad \text { and } \quad d \nu_{\widehat{\rho}}(\xi)=\sum_{i} p_{i}\left|\widehat{f}_{i}(\xi)\right|^{2} d^{*} \xi
$$

By (30), $F_{n}(\lambda)=C_{n} \lambda^{n / 2}$ and the spectral entropy is

$$
\begin{aligned}
S_{\lambda}(\rho) & =\tau\left(\ln \left(F_{n}(\Delta)\right) \rho\right)=\sum_{i} p_{i}\left\langle\ln \left(C_{n} \Delta^{n / 2}\right) f_{i}, f_{i}\right\rangle \\
& =\sum_{i} p_{i} \int_{\mathbb{R}^{n}} \ln \left(C_{n}\|\xi\|^{n}\right)\left|\widehat{f}_{i}(\xi)\right|^{2} d^{*} \xi \\
& =\int_{\mathbb{R}^{n}} \ln \left(\operatorname{vol}^{*}\left(B_{n}(0,\|\xi\|)\right) d \nu_{\widehat{\rho}}(\xi) .\right.
\end{aligned}
$$

Hence the entropy bound (17) writes here

$$
\int_{\mathbb{R}^{n}} \ln \left(\frac{d \nu_{\rho}}{d x}\right) d \nu_{\rho}(x) \leq \int_{\mathbb{R}^{n}} \ln \left(\operatorname{vol}^{*}\left(B_{n}(0,\|\xi\|)\right) d \nu_{\widehat{\rho}}(\xi)+\tau(\rho)\left(3+\ln \|\rho\|_{\infty}\right) .\right.
$$

To study the general sharpness of this bound, we first observe it implies a log-Sobolev inequality. Indeed, Jensen inequality yields

$$
\begin{aligned}
\int_{\mathbb{R}^{n}} \ln \left(\frac{d \nu_{\rho}}{d x}\right) \frac{d \nu_{\rho}(x)}{\tau(\rho)} & \leq \ln F_{n}\left(\int_{\mathbb{R}^{n}}\|\xi\|^{2} \frac{d \nu_{\widehat{\rho}}(\xi)}{\tau(\rho)}\right)+3+\ln \|\rho\|_{\infty} \\
& =\ln F_{n}\left(\frac{\mathcal{E}(\rho)}{\tau(\rho)}\right)+3+\ln \|\rho\|_{\infty} .
\end{aligned}
$$


This in turn implies a Berezin-Li-Yau type inequality for confined states in finite measure sets $\Omega$. Namely the convexity of $y \mapsto y \ln y$ leads to

$$
\tau(\rho) \leq \mu(\Omega)\|\rho\|_{\infty} e^{3} F_{n}\left(\frac{\mathcal{E}(\rho)}{\tau(\rho)}\right)
$$

This may be compared to (6) where $\varphi_{\Omega}(y)=\frac{n}{n+2} y F_{n}^{-1}\left(\frac{y}{\mu(\Omega)}\right)$ gives

$$
\tau(\rho) \leq \mu(\Omega)\|\rho\|_{\infty} F_{n}\left(\frac{n+2}{n} \frac{\mathcal{E}(\rho)}{\tau(\rho)}\right)
$$

As recalled in $\$ 2.2$ (and also $\$ 2.4$ in a discrete setting) this inequality is sharp for all $n$, in the semiclassical limit of large energy. It is indeed sharper than (48), since

$$
F_{n}\left(\frac{n+2}{n} \lambda\right)=C_{n}\left(\frac{n+2}{n} \lambda\right)^{n / 2} \leq e F_{n}(\lambda)
$$

As a consequence, the inequality (47) is sharp except possibly for the constant 3 there, which can't be taken smaller than 1 in this generality.

Remark 4.1. We notice that (48), with $e$ instead of $e^{3}$, is also an instance of the general confined states result Theorem 1.2. Indeed when applied to $\ln F_{A}^{+}(A)$, one can use

$$
\varphi_{\ln F_{A}^{+}(A), \Omega}(y) \geq \int_{0}^{y} \ln \left(\frac{u}{\mu(\Omega)}\right) d u=y \ln \left(\frac{y}{\mu(\Omega)}\right)-y
$$

instead of the weaker (not confined) $\psi$ version (45).

4.3. Proof of Theorem 1.6. The right spectral term of the previous entropy bound (47) is associated to the level sets of the symbol $\sigma_{\Delta}(\xi)=\|\xi\|^{2}$ of the Laplacian; namely at some point $\xi_{0}$, one has $\left.B_{n}\left(0,\left\|\xi_{0}\right\|\right)\right)=\left\{\xi \mid \sigma_{\Delta}(\xi) \leq \sigma_{\Delta}\left(\xi_{0}\right)\right\}$, whose volume gives the spectral density $F_{\Delta}(\lambda)$ at the energy $\lambda=\left\|\xi_{0}\right\|^{2}$. Given a state $\rho$, one can consider more general translation invariant operators, associated to other fillings of $\mathbb{R}_{\xi}^{n}$, in order to minimize the spectral entropy term $S_{\lambda}(\rho)$. We shall proceed as follows.

Let $\sigma$ be a measurable bounded function on $\mathbb{R}_{\xi}^{n}$, and $A_{\sigma}$ be defined by

$$
\widehat{A_{\sigma}(f)}(\xi)=\sigma(\xi) \widehat{f}(\xi)
$$

Let $\Omega_{\lambda}^{\sigma}=\left\{\xi \in \mathbb{R}^{n} \mid \sigma(\xi) \leq \lambda\right\}$. The spectral projection $\Pi_{A_{\sigma}}(\lambda)$ acts through Fourier transform by multiplication by $\chi_{\Omega_{\lambda}}$ and, following e.g. [[7], §4.1, §4.2], the spectral density of $A_{\sigma}$ is

$$
F_{A_{\sigma}}^{+}(\lambda)=\left\|k_{\Pi_{A_{\sigma}}(\lambda)}\right\|_{L_{x}^{2}}^{2}=\left\|\chi_{\Omega_{\lambda}^{\sigma}}\right\|_{L_{\xi}^{2}}^{2}=\operatorname{vol}^{*}\left(\Omega_{\lambda}^{\sigma}\right)
$$


This leads to the following expression for the spectral entropy of a state $\rho$, as long these integral have finite positive parts,

$$
\begin{aligned}
S_{A_{\sigma}}(\rho) & =\tau\left(\ln F_{A_{\sigma}}^{+}\left(A_{\sigma}\right) \rho\right)=\int_{\mathbb{R}} \ln \left(\operatorname{vol}^{*}\left(\Omega_{\lambda}^{\sigma}\right)\right) d \tau\left(\Pi_{A_{\sigma}}(\lambda) \rho\right) \\
& =\int_{\mathbb{R}} \ln \left(\operatorname{vol}^{*}\left(\Omega_{\lambda}^{\sigma}\right)\right) d \tau\left(\chi_{\left.\Omega_{\lambda}^{\sigma} \widehat{\rho}\right)}\right. \\
& =\int_{\mathbb{R}} \ln \left(\operatorname{vol}^{*}\left(\Omega_{\lambda}^{\sigma}\right)\right) d \nu_{\widehat{\rho}}\left(\Omega_{\lambda}^{\sigma}\right) \\
& \left.\left.=\int_{\mathbb{R}} \ln \left(\operatorname{vol}^{*}\left(\Omega_{\lambda}^{\sigma}\right)\right) d\left(\sigma_{*}\left(\nu_{\widehat{\rho}}\right)(]-\infty, \lambda\right]\right)\right),
\end{aligned}
$$

using the push-forward measure $\sigma_{*}\left(\nu_{\widehat{\rho}}\right)$. This yields

$$
S_{A_{\sigma}}(\rho)=\int_{\mathbb{R}^{n}} \ln \left(\operatorname{vol}^{*}\left(\Omega_{\sigma(\xi)}^{\sigma}\right)\right) d \nu_{\widehat{\rho}}(\xi) .
$$

The strong functional invariance of this entropy is clear here. It stays unchanged if replacing the symbol $\sigma$ into $f(\sigma)$ for any strictly increasing function $f$ on $\sigma\left(\mathbb{R}^{n}\right)$, as comes from $\Omega_{f(\sigma)(\xi)}^{f(\sigma)}=$ $\Omega_{\sigma(\xi)}^{\sigma}$. The following statement gives the minimum of these quantities and implies Theorem 1.6 .

Proposition 4.2. Given $\rho$, let $g=\frac{d \nu_{\widehat{\rho}}}{d^{*} \xi}$ and $F_{\widehat{\rho}}(y)=\operatorname{vol}^{*}(\{\xi \mid g(\xi)>y\})$ as in (20). Then one has

$$
S_{A_{\sigma}}(\rho) \geq S_{F}(\widehat{\rho})-\tau(\rho)=\int_{0}^{+\infty} F_{\widehat{\rho}}(y) \ln F_{\widehat{\rho}}(y) d y-\tau(\rho) .
$$

Equality holds if $\sigma$ is a decreasing regular filling of the level sets of $g$ in the following sense:

- for each $y$, there exists $\lambda$ such that

$$
\{\xi \mid g(\xi)>y\} \subset \Omega_{\lambda}^{\sigma}=\{\xi \mid \sigma(\xi) \leq \lambda\} \subset\{\xi \mid g(\xi) \geq y\} ;
$$

- for all $\lambda, \operatorname{vol}^{*}\left(\sigma^{-1}(\lambda)\right)=0$.

Equivalently, the level sets $\Omega_{\lambda}^{\sigma}$ of a regular filling $\sigma$ are the sets $\{\xi \mid g(\xi) \geq y\}$ (up to zero measure) for regular values of $\rho$, i.e. when $\operatorname{vol}^{*}\left(g^{-1}(y)\right)=0$, while on $g^{-1}(y)$ for the (discrete) non-regular values, they interpolate continuously in measure between $\{\xi \mid g(\xi)>y\}$ and $\{\xi \mid g(\xi) \geq y\}$. This can be achieved since the measure has no atom.

From Proposition 4.2, we notice that the use of the Laplacian is already optimal to minimize the spectral entropy of states with spherical density; Theorems 1.5 and 1.6 are equivalent on such states. On anisotropic states, one advantage of (21) over (47) lies in its stronger invariance through general linear transforms $f(x) \mapsto f(A x)$ and $\rho \mapsto \rho_{A}=A \rho A^{-1}$. In such cases, one checks easily that

$$
D \nu_{\rho_{A}}(x)=|\operatorname{det} A| D \nu_{\rho}(A x) \quad \text { while } \quad D \nu_{\widehat{\rho_{A}}}(\xi)=|\operatorname{det} A|^{-1} D \nu_{\widehat{\rho}}\left({ }^{t} A^{-1} \xi\right),
$$

giving that

$$
S_{x}\left(\rho_{A}\right)=S_{x}(\rho)-\tau(\rho) \ln |\operatorname{det} A| \quad \text { while } \quad S_{F}\left(\widehat{\rho}_{A}\right)=S_{F}(\widehat{\rho})+\tau(\rho) \ln |\operatorname{det} A|,
$$

which keeps the entropy sum unchanged in (47). 
Proof of Proposition 4.9. Let $v(\xi)=\operatorname{vol}^{*}\left(\Omega_{\sigma(\xi)}^{\sigma}\right)$ and $\left.\left.F_{\sigma}(z)=v_{*}\left(\nu_{\widehat{\rho}}\right)(] 0, z\right]\right)$. Then by (49)

$$
S_{A_{\sigma}}(\rho)=\int_{0}^{+\infty} \ln y d F_{\sigma}(y)=-\int_{0}^{1} F_{\sigma}(y) \frac{d y}{y}+\int_{1}^{+\infty}\left(\tau(\rho)-F_{\sigma}(y)\right) \frac{d y}{y},
$$

by Fubini, since $F_{\sigma}(+\infty)=\tau(\widehat{\rho})=\tau(\rho)$. Hence $S_{A_{\sigma_{1}}}(\rho) \geq S_{A_{\sigma_{2}}}(\rho)$ if $F_{\sigma_{1}} \leq F_{\sigma_{2}}$, and we have to look for upper bounds for $F_{\sigma}$ to minimize $S_{A_{\sigma}}(\rho)$.

By definition, one has

$$
F_{\sigma}(y)=\int_{D_{y}^{\sigma}} g(\xi) d^{*} \xi \quad \text { with } \quad D_{y}^{\sigma}=\left\{\xi \mid \operatorname{vol}^{*}\left(\Omega_{\sigma(\xi)}^{\sigma}\right) \leq y\right\} .
$$

Clearly one has $\{\xi \mid \sigma(\xi)<\lambda\} \subset D_{y}^{\sigma} \subset \Omega_{\lambda}^{\sigma}$ where $\lambda=\sup _{D_{y}^{\sigma}} \sigma$, and thus $\operatorname{vol}^{*}\left(D_{y}^{\sigma}\right) \leq y$ with equality if $\operatorname{vol}^{*}\left(\sigma^{-1}(\lambda)\right)=0$. Hence by the 'bathtub principle' (see [5, Theorem 12.3]) one has

$$
F_{\sigma}(y) \leq F(y)=\int_{\left\{g>F_{\widehat{\rho}}^{-1}(y)\right\}} g(\xi) d^{*} \xi+F_{\widehat{\rho}}^{-1}(y)\left(y-F_{\widehat{\rho}}\left(F_{\widehat{\rho}}^{-1}(y)\right)\right),
$$

with $F_{\widehat{\rho}}^{-1}(y)=\inf \left\{z \mid F_{\widehat{\rho}}(z) \leq y\right\}$. Indeed, this comes from the identity

$$
\begin{aligned}
F_{\sigma}(y)-F(y)=\int_{D_{y}^{\sigma} \cap\left\{g \leq F_{\widehat{\rho}}^{-1}(y)\right\}}\left(g(\xi)-F_{\widehat{\rho}}^{-1}(y)\right) d^{*} \xi & \\
& \quad-\int_{\left(D_{y}^{\sigma}\right)^{c} \cap\left\{g>F_{\widehat{\rho}}^{-1}(y)\right\}}\left(g(\xi)-F_{\widehat{\rho}}^{-1}(y)\right) d^{*} \xi+F_{\widehat{\rho}}^{-1}(y)\left(\operatorname{vol}^{*}\left(D_{y}^{\sigma}\right)-y\right) .
\end{aligned}
$$

Moreover this shows that equality holds in (51) if $\operatorname{vol}^{*}\left(D_{y}^{\sigma}\right)=y$ and, up to zero measure sets, $\left\{g>F_{\widehat{\rho}}^{-1}(y)\right\} \subset D_{y}^{\sigma} \subset\left\{g \geq F_{\widehat{\rho}}^{-1}(y)\right\}$; which is fulfilled for regular fillings by the discussion above.

We rewrite the function $F(y)$ in a more convenient form. Since $\tau(\rho)=\tau(\widehat{\rho})=\int_{\mathbb{R}^{n}} g(\xi) d^{*} \xi$, one has

$$
\begin{aligned}
\int_{\left\{g>F_{\widehat{\rho}}^{-1}(y)\right\}} g(\xi) d^{*} \xi & =\tau(\rho)-\int_{\left\{g \leq F_{\widehat{\rho}}^{-1}(y)\right\}} g(\xi) d^{*} \xi \\
& =\tau(\rho)-\int_{\left\{0 \leq u<g(\xi) \leq F_{\widehat{\rho}}^{-1}(y)\right\}} d u d^{*} \xi \\
& =\tau(\rho)-\int_{0}^{F_{\widehat{\rho}}^{-1}(y)}\left(F_{\widehat{\rho}}(u)-F_{\widehat{\rho}}\left(F_{\widehat{\rho}}^{-1}(y)\right) d u\right. \\
& =\tau(\rho)-\int_{0}^{F_{\widehat{\rho}}^{-1}(y)} F_{\widehat{\rho}}(u) d u+F_{\widehat{\rho}}^{-1}(y) F_{\widehat{\rho}}\left(F_{\widehat{\rho}}^{-1}(y)\right) .
\end{aligned}
$$

Then by (51),

$$
\begin{aligned}
F(y) & =\tau(\rho)-\int_{0}^{F_{\widehat{\rho}}^{-1}(y)}\left(F_{\widehat{\rho}}(u)-y\right) d u \\
& =\tau(\rho)-\int_{\left\{y<v<F_{\widehat{\rho}}(u)\right\}} d v d u,
\end{aligned}
$$


since by right continuity of $F_{\widehat{\rho}}$, one has $u<F_{\widehat{\rho}}^{-1}(y)$ iff $F_{\widehat{\rho}}(u)>y$. Therefore

$$
F(y)=\tau(\rho)-\int_{y}^{+\infty} F_{\widehat{\rho}}^{-1}(v) d v=\int_{0}^{y} F_{\widehat{\rho}}^{-1}(v) d v
$$

since

$$
\begin{aligned}
\tau(\rho) & =\int_{\mathbb{R}^{n}} g(\xi) d^{*} \xi=\int_{\{0 \leq u<g(\xi)\}} d u d^{*} \xi=\int_{0}^{+\infty} F_{\widehat{\rho}}(u) d u \\
& =\int_{\left\{0 \leq v<F_{\widehat{\rho}}(u)\right\}} d v d u=\int_{0}^{+\infty} F_{\widehat{\rho}}^{-1}(v) d v .
\end{aligned}
$$

Finally, (50) and (52) lead to

$$
\begin{aligned}
S_{A_{\sigma}}(\rho) & \geq \int_{0}^{+\infty} \ln y F_{\widehat{\rho}}^{-1}(y) d y=\int_{\left\{0<z<F_{\widehat{\rho}}^{-1}(y)\right\}} \ln y d z d y \\
& =\int_{\left\{0<y<F_{\widehat{\rho}}(z)\right\}} \ln y d y d z \\
& =\int_{0}^{+\infty} F_{\widehat{\rho}}(z)\left(\ln F_{\widehat{\rho}}(z)-1\right) d z=S_{F}(\widehat{\rho})-\tau(\rho),
\end{aligned}
$$

as claimed in Proposition 4.2 .

4.4. Proof of Corollary 1.7. We deduce Corollary 1.7 from Theorem 1.6. This relies on the following entropy comparison:

$$
S_{F}(\widehat{\rho}) \leq S_{\xi}(\widehat{\rho})+\tau(\rho)(1+\ln \tau(\rho)) .
$$

Indeed, one has

$$
\begin{aligned}
-S_{\xi}(\widehat{\rho})-\tau(\rho) & =\int_{\mathbb{R}^{n}} \ln \left(\frac{d \nu_{\widehat{\rho}}}{d^{*} \xi}\right) d \nu_{\widehat{\rho}}-\tau(\widehat{\rho}) \\
& =\int_{\left\{0<y<\frac{d \nu_{\widehat{\rho}}}{d^{*} \xi}\right\}} \ln y d y d^{*} \xi \\
& =\int_{0}^{+\infty} F_{\widehat{\rho}}(y) \ln y d y,
\end{aligned}
$$

thus

$$
\begin{aligned}
S_{F}(\widehat{\rho})-S_{\xi}(\widehat{\rho})-\tau(\rho) & =\int_{0}^{+\infty} \ln \left(y F_{\widehat{\rho}}(y)\right) F_{\widehat{\rho}}(y) d y \\
& \leq \int_{0}^{+\infty} \ln (\tau(\rho)) F_{\widehat{\rho}}(y) d y=\tau(\rho) \ln \tau(\rho),
\end{aligned}
$$

since $y F_{\widehat{\rho}}(y)=y \operatorname{vol}^{*}\left\{\xi \mid \frac{d \nu_{\widehat{\rho}}}{d^{*} \xi}(\xi)>y\right\} \leq \int_{\mathbb{R}^{n}} \frac{d \nu_{\widehat{\rho}}}{d^{*} \xi} d^{*} \xi=\tau(\rho)$. Then, (21) and (53) give

$$
S_{x}(\rho)+S_{\xi}(\widehat{\rho}) \geq-\tau(\rho)\left(3+\ln \tau(\rho)+\ln \|\rho\|_{\infty}\right) .
$$

Then we observe that, except for the term $-3 \tau(\rho)$, this expression is additive in taking tensor product of unit trace states. Therefore, applying it to $\otimes^{N} \rho$ on $\mathbb{R}^{n N}$, and dividing by $N$ for $N \nearrow+\infty$, gives (22) on unit trace states, and the general statement by homogeneity. 
4.5. Around Corollary 1.7. It is appealing trying to express, or bound, the right side of (22) using von Neumann's entropy of $\rho$. Following [8, 9] this intrisic entropy is defined for unit trace states by

$$
S(\rho)=-\tau(\rho \ln \rho) .
$$

For such states, one has $S(\rho) \geq-\ln \|\rho\|_{\infty}$, with equality on normalized projections on finite dimensional spaces $\rho=\Pi_{V} / \operatorname{dim} V$. Hence on these projections, (22) reads

$$
S_{x}(\rho)+S_{\xi}(\widehat{\rho}) \geq S(\rho)(=\ln \operatorname{dim} V) .
$$

We don't know whether this holds for general unit trace states. An interesting family of examples here is given by the heat of the harmonic oscillator, which is the semigroup

$$
\rho_{t}=\exp \left(-t\left(\Delta+\|x\|^{2}\right)\right)
$$

acting on $L^{2}\left(\mathbb{R}_{x}^{n}\right)$. This state is the $n$-th tensor product of the one-dimensional case. Furthermore it is self-dual in Fourier transform, i.e. $\widehat{\rho}_{t}=\rho_{t}$. The kernel of $\rho_{t}$ is given by Mehler's formula (see [1], Chap. 4.2]):

$$
\rho_{t}(x, y)=(2 \pi \sinh 2 t)^{-n / 2} \exp \left(-\frac{1}{2}(\operatorname{coth} 2 t)\left(\|x\|^{2}+\|y\|^{2}\right)+(\sinh 2 t)^{-1}\langle x, y\rangle\right),
$$

so that its density and trace are

$$
\frac{d \nu_{\rho_{t}}}{d x}=\rho_{t}(x, x)=(2 \pi \sinh 2 t)^{-n / 2} \exp \left(-(\tanh t)\|x\|^{2}\right) \quad \text { and } \quad \tau\left(\rho_{t}\right)=(2 \sinh t)^{-n} .
$$

This leads easily to the entropies of the normalized states $\lambda_{t}=\rho_{t} / \tau\left(\rho_{t}\right)$, namely

$$
S_{x}\left(\lambda_{t}\right)=\frac{n}{2}-\frac{n}{2} \ln \left(\frac{\tanh t}{\pi}\right) \quad \text { while } \quad S_{\xi}\left(\widehat{\lambda_{t}}\right)=\frac{n}{2}-\frac{n}{2} \ln \left(\frac{\tanh t}{\pi}\right)-n \ln (2 \pi),
$$

hence

$$
S_{x}\left(\lambda_{t}\right)+S_{\xi}\left(\widehat{\lambda}_{t}\right)=n-n \ln 2-n \ln (\tanh t) .
$$

To compute von Neumann's entropy of $\lambda_{t}$, we recall that on $\mathbb{R}$, the spectrum of $\rho_{t}$ is given by $p_{k}=e^{-(2 k+1) t}, k \in \mathbb{N}$. One finds that

$$
\begin{aligned}
S\left(\lambda_{t}\right) & =n S\left(\lambda_{t}^{\mathbb{R}}\right)=-n \sum_{k \geq 0}(2 \sinh t) p_{k} \ln \left((2 \sinh t) p_{k}\right) \\
& =-n \ln (2 \sinh t)-2 n t \sinh t \sum_{k \geq 0}(-2 k-1) e^{-(2 k+1) t} \\
& =-n \ln (2 \sinh t)-2 n t \sinh t\left(\frac{1}{2 \sinh t}\right)^{\prime} \\
& =n t \operatorname{coth} t-n \ln 2-n \ln (\sinh t)
\end{aligned}
$$

Therefore we obtain

$$
S_{x}\left(\lambda_{t}\right)+S_{\xi}\left(\widehat{\lambda}_{t}\right)-S\left(\lambda_{t}\right)=n(1+\ln (\cosh t)-t \operatorname{coth} t),
$$

which is easily seen to be increasing in $t$ and positive. Hence these states also satisfy the entropy bound (54), sharply when $t$ goes to 0 . 


\section{REFERENCES}

[1] N. Berline, E. Getzler, and M. Vergne. Heat kernels and Dirac operators, volume 298 of Grundlehren der Mathematischen Wissenschaften [Fundamental Principles of Mathematical Sciences]. Springer-Verlag, Berlin, 1992.

[2] T. Coulhon. Ultracontractivity and Nash type inequalities. J. Funct. Anal., 141(2):510-539, 1996.

[3] E. B. Davies. Heat kernels and spectral theory, volume 92 of Cambridge Tracts in Mathematics. Cambridge University Press, Cambridge, 1989.

[4] P. Li and S. T. Yau. On the Schrödinger equation and the eigenvalue problem. Comm. Math. Phys., 88(3):309-318, 1983.

[5] E. H. Lieb and M. Loss. Analysis, volume 14 of Graduate Studies in Mathematics. American Mathematical Society, Providence, RI, second edition, 2001.

[6] E. H. Lieb and W. E. Thirring. Inequalities for the moments of the eigenvalues of the Schrödinger Hamiltonian and their relation to Sobolev inequalities. Stud. math. Phys., Essays Honor Valentine Bargmann, 269-303 (1976)., 1976.

[7] M. Rumin. Spectral density and Sobolev inequalities for pure and mixed states. To appear in Geom. Funct. Anal., 2010. Preprint.

[8] J. von Neumann. Mathematical foundations of quantum mechanics. Princeton Landmarks in Mathematics. Princeton University Press, Princeton, NJ, 1996. Translated from the German and with a preface by Robert T. Beyer, Twelfth printing, Princeton Paperbacks.

[9] Wikipedia. Density matrix. <http://en.wikipedia.org/wiki/Density_matrix >.

Laboratoire de Mathématiques d'Orsay, UMR 8628, CNRS et Université Paris-Sud, 91405 Orsay, France

E-mail address: michel.rumin@math.u-psud.fr 